\title{
APORTE NUTRICIONAL DE DESAYUNOS EXPENDIDOS EN VENTA AMBU- LANTE ALREDEDOR DE UNA UNIVERSIDAD DE LIMA, 2018
}

\section{NUTRITIONAL SUPPLY IN STREET SOLD BREAKFASTS AROUND A UNI- VERSITY IN LIMA, 2018}

\author{
MARIA LUISA AROTINCO GARCÍA \\ Universidad Norbert Wiener \\ JoHANnA DEl CARMEn León CÁCERES \\ Universidad Norbert Wiener \\ JoSE JAIRo NARREA VARGAS \\ Universidad Norbert Wiener \\ Michelle FATIMA LOZADA-URBANO \\ Universidad Norbert Wiener
}

\section{RESUMEN}

La nutrición tiene un papel preponderante en la salud mental y física. El desayuno permite un mejor rendimiento y mayor grado de concentración. En la actualidad, los jóvenes universitarios adoptan prácticas alimentarias dependientes del acceso al mismo, por lo que muchos consumen el desayuno que se venden en los alrededores de la universidad. El presente estudio evaluó el aporte nutricional de desayunos a nivel de macronutrientes y calorías que ofrece la venta ambulante cerca de una universidad de Lima. El estudio es de tipo descriptivo-transversal. La muestra se obtuvo de cuatro puntos de venta de ambulante, durante los meses de junio a julio del 2018. El aporte nutricional de los desayunos expendidos en los puntos de venta de la zona alrededor de la universidad muestra preparaciones hipocalóricas con 192,3 - $370 \mathrm{Kcal}(13,2-15,7 \%)$, respecto al requerimiento calórico promedio propuesto de $400-500 \mathrm{Kcal}(20-25 \%)$, en una población urbana promedio que consume 2000 Kcal al día.

PALABRAS CLAVE: desayunos, macronutrientes, universitarios. 
Nutrition plays a major role in mental and physical health. Breakfast allows a better performance and a higher degree of concentration. Currently, young university students adopt food practices that depend on the access to it, which is why many consume the breakfast sold around the university. The present study evaluated the nutritional contribution of breakfasts at the level of macronutrients and calories offered by the street sale near a university in Lima. This is a descriptive-cross-sectional study. The sample was obtained from four points of sale of itinerant, during the months of June to July 2018. The nutritional contribution of the breakfasts sold in the points of sale in the area around the university shows hypocaloric preparations with 192,3 - $370 \mathrm{Kcal}(13,2$ $-15,7 \%$ ), with respect to the proposed average caloric requirement of 400-500 Kcal (20$25 \%$ ), in an average urban population that consumes $2000 \mathrm{Kcal}$ per day.

KEY WORDS: breakfast, macronutrients, university students. 


\section{INTRODUCCIÓN}

La alimentación juega un rol importante en la salud mental y física (1). La ingesta de la primera comida en el día, o desayuno, ha sido estudiada a lo largo de los últimos años; se reconoce su importancia para cubrir las necesidades energéticas tempranas, además de su relación positiva ante la función cognitiva, particularmente en la memoria. Se sabe que debería aportar el $20-25 \%$ de las necesidades energéticas diarias y una proporción equilibrada de carbohidratos, proteínas y grasas, con el fin de permitir un adecuado rendimiento físico e intelectual. Adicionalmente, el consumo del desayuno se asocia con menos depresión (2) y sensación de felicidad (3). La omisión del mismo podría causar fatiga y alteración en la concentración requerida para las tareas diarias, así como un impacto negativo en el aprendizaje de estudiantes universitarios (4).

La población peruana tiende a llevar una vida agitada por motivos de trabajo y estudio, es posible que por esa razón omitan el desayuno, lo consuman fuera de casa o consuman un desayuno de forma rápida «al paso»; es aquí donde los centros de expendio de alimentos como los quioscos o las carretillas se presentan con mayor protagonismo para esta comida. Además de ello, es frecuente el acceso a estos centros de venta, como resultado de adaptación a un estilo de vida universitaria $(5,6)$.

Es importante determinar y evaluar el contenido nutricional de los desayunos expendidos en venta ambulante, aun cuando no necesariamente integran alimentos procesados; se sabe que integran alimentos oriundos del Perú como la quinua, maca y soya, que se caracterizan por contener proteínas de alto valor nutricional. La quinua, por ejemplo, es el único alimento vegetal que posee todos los aminoácidos esenciales, oligoelementos, vitaminas y no contiene gluten; los aminoácidos esenciales se encuentran en el núcleo del grano, a diferencia de otros cereales que los tienen en la cáscara, como el arroz o trigo (7). La maca contiene proteínas, minerales, ácidos grasos insaturados, fibra y también aporta energía. La soya tiene un importante contenido de proteínas, vitaminas y minerales que favorece el crecimiento y desarrollo de los adolescentes; también es rica en lisina, que aporta al desarrollo de las células cerebrales, fortaleciendo la memoria y facilitando el aprendizaje (8).

Los estudios de desayunos ofrecidos en puestos de venta ambulatoria en el cercado de Lima presentaron alta densidad Revista de Investigación de la Universidad Norbert Wiener, 2020, N.॰ 9 
energética pero baja densidad de nutrientes; estos incluyeron cereales como la quinua y la soya con adicionales como el pan con tortilla, pan con palta y pan con camote (9). Otros estudios que valoraron la calidad nutricional de desayunos en estudiantes universitarios de la Ciudad de México asociaron a una mayor calidad nutricional cuando integran frutas, verduras, granos sin grasa y alimentos fuente de proteína animal, mientras que los desayunos con menor calidad fueron ricos en azúcar y alimentos sin calorías (10).

Pocos estudios han identificado y analizado la composición química-nutricional de los desayunos que se venden de forma ambulante. En ese sentido, se determinó el aporte y adecuación nutricional de desayunos vendidos en una universidad de Lima.

\section{Metodología}

\section{REQUERIMIENTOS}

El requerimiento calórico para satisfacer el gasto energético total diario ponderal según la actividad para la población peruana urbana es 1867,4 a $2003 \mathrm{Kcal}$ (11).

\section{AdecuAción}

La ingesta del desayuno debe represen-

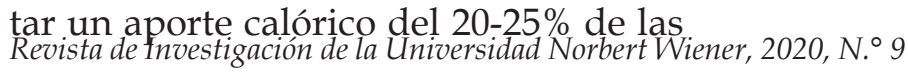

calorías ingeridas totales en un día. $\mathrm{Al}$ considerar una ingesta promedio de 2000 Kcal por día, un desayuno deberá aportar un rango de 400 a $500 \mathrm{Kcal}$. Los puntos de corte para la interpretación del porcentaje de adecuación calórica son: muy baja: $<75 \%$ ( $<300 \mathrm{Kcal}$ ), adecuación baja: 75-89 \% (300-356 Kcal), adecuado: $90-110 \%$ (360 - $440 \mathrm{Kcal})$ y sobre adecuación: >110 \% (>440 Kcal) (11).

\section{MATERIALES Y MÉTODOS}

El estudio es tipo descriptivo-transversal. La muestra se obtuvo de cuatro puntos de venta ambulante ubicados alrededor de una universidad de Lima en el distrito de Cercado de Lima, Perú.

Para la recolección de muestras se compraron las bebidas y los sándwiches por separado en distintos días durante el periodo de junio y julio del 2018, en una cantidad de tres unidades por cada preparación. En cuanto a la valoración del contenido de las preparaciones líquida o bebidas expendidas se realizó mediante una entrevista directa y auto-referencia de los encargados del puesto ambulante o dueños de los módulos, se les preguntó sobre la preparación e ingredientes utilizados en cada preparación, utilizando la valoración por medida casera o gramaje. Para los alimen- 
tos sólidos, se compraron de igual forma tres unidades de cada preparación, que se trasladaron inmediatamente al laboratorio de dietética y se aplicó el peso directo utilizando una balanza digital con precisión en gramos con capacidad máxima de $2 \mathrm{~kg}$; para determinar la composición química-nutricional se utilizó la tabla de composición química de alimentos peruanos y la tabla de factor de conversión de cocido a crudo.

\section{RESULTADOS}

La tabla 1 presenta las preparaciones sólidas de alimentos y composición química-nutricional que integran los desayunos expendidos en los puestos de venta ambulante, del cual se puede observar que los puestos de venta presentan preparaciones con los mismos alimentos en diferentes gramajes.

Tabla 1. Contenido químico-nutricional de las preparaciones sólidas incluidas en los desayunos obtenidas en los puestos de ambulantes

\begin{tabular}{|c|c|c|c|c|c|c|c|c|}
\hline \multirow[t]{2}{*}{ Tipos de preparación } & \multicolumn{2}{|c|}{ Peso (g) } & \multicolumn{2}{|c|}{ Carbohidrato } & \multicolumn{2}{|c|}{ Proteína (g) } & \multicolumn{2}{|c|}{ Lípido (g) } \\
\hline & $\mathrm{X}$ & $\overline{\mathrm{DS}}$ & $\mathrm{X}$ & $\overline{\mathrm{DS}}$ & $\mathrm{X}$ & $\overline{\mathrm{DS}}$ & $\mathrm{X}$ & $\overline{\mathrm{DS}}$ \\
\hline \multicolumn{9}{|l|}{ Puesto ambulante 1} \\
\hline Pan con pollo & 43,0 & 4,0 & 18,2 & 1,4 & 4,6 & 0,0 & 0,3 & 0,0 \\
\hline Pan con palta & 89,0 & 2,0 & 22,2 & 0,0 & 3,5 & 0,0 & 7,4 & 0,4 \\
\hline Pan con huevo frito & 67,6 & 8,4 & 19,6 & 0,0 & 7,7 & 2,2 & 16,4 & 0,6 \\
\hline Pan con queso & 69,0 & 2,0 & 20,2 & 0,0 & 9,3 & 0,4 & 7,8 & 0,0 \\
\hline \multicolumn{9}{|l|}{ Puesto ambulante 2} \\
\hline Pan con palta & 71,0 & 3,0 & 24,2 & 0,0 & 3,8 & 0,0 & 4,3 & 0,0 \\
\hline Pan con queso & 79,0 & 2,0 & 25,6 & 0,0 & 14,9 & 0,8 & 8,4 & 0,0 \\
\hline \multicolumn{9}{|l|}{ Puesto ambulante 3} \\
\hline Pan con palta & 92,0 & 3,0 & 31,4 & 0,0 & 4,3 & 0,0 & 5,8 & 0,0 \\
\hline Pan con queso & 81,0 & 2,0 & 27,0 & 0,0 & 10,4 & 0,0 & 8,1 & 0,1 \\
\hline Pan con huevo frito & 74,0 & 4,6 & 19,6 & 0,8 & 7,7 & 0,2 & 16,4 & 0,0 \\
\hline \multicolumn{9}{|l|}{ Puesto ambulante 4} \\
\hline Pan con pollo & 43,0 & 4,0 & 23,3 & 1,0 & 3,4 & 0,4 & 0,7 & 0,0 \\
\hline Pan con palta & 89,0 & 3,0 & 22,2 & 0,0 & 8,8 & 0,0 & 7,4 & 0,2 \\
\hline Pan con huevo frito & 87,0 & 3,4 & 22,1 & 0,0 & $\mathrm{X}$ & 1,0 & 44,2 & 0,2 \\
\hline
\end{tabular}


Aporte nutricional de desayunos expendidos en venta ambulante alrededor de una universidad de lima, 2018

La tabla 2 presenta las preparaciones se observa que utilizan el mismo grlíquidas o bebidas y composición quími- amaje para su preparación en todos los ca de que integran los desayunos expen- puestos ambulantes.

didos en los puestos ambulantes, donde

Tabla 2. Contenido químico-nutricional de las preparaciones líquidas incluidas en los desayunos obtenidas en los puestos de ambulantes

\begin{tabular}{|c|c|c|c|c|c|c|c|c|}
\hline \multirow[t]{2}{*}{ Preparación } & \multicolumn{2}{|c|}{ Volumen (ml9 } & \multicolumn{2}{|c|}{ Carbohidrato (g) } & \multicolumn{2}{|c|}{ Proteína (g) } & \multicolumn{2}{|c|}{ Lípido (g) } \\
\hline & $\mathrm{X}$ & DS & $\mathrm{X}$ & DS & $\mathrm{X}$ & DS & $X$ & DS \\
\hline Quinua (bebida) & 200 & 0,0 & 23,3 & 0,0 & 17,0 & 0,0 & 0,7 & 0,0 \\
\hline Maca (bebida) & 200 & 0,0 & 31,6 & 0,0 & 2,9 & 0,0 & 0,4 & 0,0 \\
\hline Soya (bebida) & 200 & 0,0 & 20,6 & 0,0 & 4,5 & 0,0 & 3,0 & 0,0 \\
\hline
\end{tabular}

En la tabla 3 se observa el valor calóri- das las probabilidades de consumo que co total de cada desayuno compuesto integran una preparación sólida con por una preparación líquido y sólida una preparación líquida para un mismo (bebida y sándwich), considerando to- puesto de venta ambulante.

Tabla 3. Valor calórico promedio de desayunos

\begin{tabular}{ccccc}
\hline & \multicolumn{4}{c}{ Energía (Kcal) } \\
\hline prom. & mín. & máx. & DS \\
\hline Desayuno 1 & 263,9 & 192,3 & 345,7 & 49,6 \\
Desayuno 2 & 288,9 & 247,5 & 337,5 & 38,5 \\
Desayuno 3 & 313,7 & 274,3 & 347,0 & 26,3 \\
Desayuno 4 & & & & \\
& 278,7 & 204,1 & 370,0 & 63,3 \\
\hline
\end{tabular}


Los resultados presentados en la tabla considerando todas las probabilidades 4 corresponden al porcentaje de ade- de consumo que integran una prepacuación calórica que representa cada de- ración. Para todos los casos se considersayuno compuesto por una preparación aron una adecuación calórica porcentulíquida y sólida (bebida y sándwich),- al respecto a una dieta de 2000 Kcal.

Tabla 4. Porcentaje de adecuación calórica de cada desayuno

\begin{tabular}{ccccc}
\hline & \multicolumn{4}{c}{ Índice de adecuación calórica (\%) } \\
\hline prom. & mín. & máx. & DS \\
\hline Desayuno 1 & 13,2 & 9,6 & 17,3 & 2,5 \\
Desayuno 2 & 14,4 & 12,4 & 16,9 & 1,9 \\
Desayuno 3 & 15,7 & 13,7 & 17,4 & 1,3 \\
Desayuno 4 & 13,9 & 10,2 & 18,5 & 3,2 \\
\hline
\end{tabular}

Discusión

Nuestros hallazgos demuestran que las preparaciones, considerando una unidad de bebida y sándwich, que se venden en los puestos ambulantes en los alrededores de una universidad en Lima se encuentran dentro de un rango disminuido para el aporte calórico promedio para una población de jóvenes. En relación al contenido químico-nutricional de las preparaciones sólidas (sándwich) el mayor aporte de carbohidrato es de 31,4 g $(125,6 \mathrm{Kcal})$ por par- te de la preparación pan con palta, el mayor aporte de proteína es de $14,9 \mathrm{~g}$ (59,6 Kcal) por parte de la preparación pan con queso y el mayor aporte de lípidos es de 16,4 g (147,6 Kcal) por parte de la preparación pan con huevo frito. En cuanto a las preparaciones líquidas (bebidas), el mayor aporte de carbohidrato es de 31,6 g (126,4 Kcal) por parte de la bebida de la maca, el mayor aporte de proteína es de $17 \mathrm{~g}$ (68 Kcal) por parte de la bebida de quinua y el mayor aporte de lípidos es de $3 \mathrm{~g}$ ( $27 \mathrm{Kcal}$ ) por parte de la bebida de soya.

Revista de Investigación de la Universidad Norbert Wiener, 2020, N. 9 
Si bien es cierto que el costo es relativamente económico si se compara con lo que se ofrece en otros lugares, como el caso de los desayunos expendidos en las tiendas TAMBO® o REPSOL $®$ (ambos situados alrededor de la universidad) que también ofrecen una preparación compuesta por una bebida de avena como puede ser el refresco Actiavena de la marca Gloria ${ }^{\circledR}$ junto a un sándwich de queso y jamón de la marca Andree®, con un precio que llega a ser tres veces mayor al de los puestos ambulantes, los del segundo caso resultan de fácil acceso y la combinación de los productos pueden ser bastante atractivos para el consumidor.

Es precisamente la gran cantidad de desayunos vendidos en puestos ambulantes lo que motivó a investigar cuál es la composición nutricional de estas preparaciones. Sin embargo, después de nuestro análisis, los desayunos proveen una cantidad disminuida en energía cerca a lo normo-calórico. Estos resultados incluyen una unidad de preparación sólida y líquida; si se consume más de una unidad, los valores energéticos cambiarían considerablemente. La valoración para la población universitaria está referida a una unidad, lo que indica que este consumo es hipocalórico.
En cuanto al aporte de macronutrientes, de los cuatro puestos evaluados el mayor aporte proviene de los carbohidratos. Esto podría incrementarse en ocasiones, ya que en las bebidas agregan harina o maicena para poder espesar, además pueden llegar a tener en su composición una gran cantidad de azúcar. En cuanto a la cantidad de nutrientes, estudios como el de Huapaya, donde se valoró los desayunos ofrecidos en puestos de venta ambulatorios, llegaron a la conclusión que los nutrientes no son adecuados; sin embargo presentan alta densidad energética (9).

En referencia a un estudio en Guatemala, que mostró desayunos comprados en vendedores de la calle, en 100 gramos, con una mediana del valor nutricional del desayuno de 127,5 Kcal, este producto concierne al patrón de consumo de los pobladores de Guatemala. Lo mismo ocurre Lima, ya que los desayunos de este tipo son muy frecuentes en muchos departamentos del Perú y en muchos distritos de Lima, y se parecen a los hallazgos obtenidos por Vargas, quien describe alimentación insuficiente en cantidad y en nutrientes en estudiantes universitarios en Colombia (12). Sin embargo, aunque en nuestro estudio los desayunos fueron hipocalóricos a diferencia de los desayunos descritos por Monroy-Valle (13), en Guatemala 
mostraron altas cantidades de carbohidratos refinados, grasas saturadas y poca proteína.

Se recomienda para futuras investigaciones incluir medidas desde la ingesta dietética de los consumidores frecuentes o con gran acceso a estos puestos de venta ambulante hasta la valoración del componente microbiológico para valorar la seguridad de su consumo.

\section{CONCLUSión}

El aporte calórico de los desayunos expendidos en los puntos de venta ambulante alrededor de una universidad de Lima son hipocalóricos, por lo que se encuentran dentro del rango de adecuación calórica baja $(13,2 \%$ a $15,7 \%)$, considerando una adecuación normal de $20 \%$ a $25 \%$ respecto a una ingesta promedio de $2000 \mathrm{Kcal}$ por día.

Un agradecimiento a los estudiantes Anita Luis Chávez Bernal, Patricia Márquez Roca, Yanely Rosales Castillo, por su apoyo en la obtención de la muestra.

\section{REFERENCIAS BIBLIOGRÁFICAS}

1. O'Neil A, Quirk SE, Housden S, Brennan SL, Williams LJ, Pasco JA, et al. (2014). Relationship between diet and mental health in children and adolescents: a systematic review. Am J Public Health 104(10): 31-42.

2. Rampersaud GC, Pereira MA, Girard BL, Adams J, Metzl JD. (2005). Breakfast habits, nutritional status, body weight, and academic performance in children and adolescents. J Am Diet Assoc 105(5):743-760.

3. Lesani A, Mohammadpoorasl A, Javadi M, Esfeh JM, Fakhari A (2016). Eating breakfast, fruit and vegetable intake and their relation with happiness in college students. Eat Weight Disord. 21(4):645-651.

4. Sámano R, Hernández-Chávez C, Chico-Barba G, et al. (2019). Breakfast Nutritional Quality and Cognitive Interference in University Students from Mexico City. Int J Environ Res Public Health. 16(15):267.

5. Laska MN, Hearst MO, Forsyth A, Pasch KE, Lytle L. (2010). Neighbourhood food environments: are they associated with adolescent dietary intake, food purchases and weight status? Public Health Nutr. 13(11):1757-1763.

6. He M, Tucker P, Gilliland J, Irwin JD, Larsen K, Hess P. (2012). The influence of local food environments on adolescents' food purchasing behaviors. Int J Environ Res Public Health. 9(4):1458-1471.

7. Alvarado J. Principios de Ingeniería Aplicados a los Alimentos. Edit. Radio 
Comunicaciones, División de artes Gráficas. Quito-Ecuador. 1996. Pp.109-118.

8. Cerón LE. Proyecto sobre fomento del cultivo de quinua en Perú. Primera mesa redonda sobre investigación de la quinua en Perú, (1976). ICBF, Lima.

9. Huapaya E J, Vera R. Calidad de nutrición de los desayunos ofrecidos en los puestos ambulatorios y nivel de conocimiento sobre la alimentación de sus vendedores en el Cercado

de Lima. Nutrición pública, 2011. Disponible en: www. iidenut.org/pdf_revista_tec.../ RENUT\%202011\%20TEC_15_788796.pdf

10. Sámano R, Hernández-Chávez C, Chico-Barba G, et al. (2019). Breakfast Nutritional Quality and Cognitive Interference in University Students from Mexico City. Int J Environ Res Public Health. 16(15):2671.

11. Centro Nacional de Alimentación y Nutrición (CENAN). Requerimientos De Energía para la Población

Peruana, 2012. Disponible en:www.ins.gob.pe/.../Requerimiento $\% 20$ de $\% 20$ energía $\% 20$ para $\% 20$ la\%20población $\% 2 \ldots$

12.. Vargas M, Becerra B, Prieto E. Evaluación de la ingesta dietética en estudiantes universitarios. Bogotá, Colombia. Salud publica vol.12 no.1 Bogotá /Feb. 2010. Disponible en: http://www.scielo.org.co/ scielo.php?script=sci_arttext\&pid=S0124- 00642010000100011 .
Toledo Chaves PF. Composición $y$ valor nutritivo de almuerzos $y$ desayunos comprados fuera del hogar en zonas urbanas de Guatemala [Composition and nutritional value of lunches and breakfasts purchased outside the households in urban Guatemala]. Nutr Hosp. 2014;31(2):908-915. Published 2014 Nov 1. DOI: 10.3305/ nh.2015.31.2.7752

\section{Monroy-Valle MM, Monroy Valle K,}

